\title{
Alocação de Canais em Sistemas de Comunicação Celular Empregando Algoritmo Genético Distribuído e SIR com Temporização
}

\author{
Ailton Akira Shinoda e Leandro Calixto T. de Albuquerque
}

\begin{abstract}
Resumo-O objetivo deste trabalho é apresentar uma proposta alternativa de alocação de canais em sistemas celulares, empregando algoritmo genético (AG), processamento paralelo e a relação sinal interfêrencia (SIR) com limitação de tempo na obtenção de um conjunto de canais, baseado na função de custo ou interferência, para satisfazer a demanda de chamadas do sistema.
\end{abstract}

Palavras-Chave-Alocação de Canais, Rede Celular, AG, SIR, Processamento Distribuído.

Abstract-The objective of this work is to show an alternative approach for the channel assignment in cellular system using genetic algorithm (GA), parallel processing and signal-interference ratio (SIR) with time limitation to get a channel set, based on cost function or interference, to attend the demand of calls on the system.

Keywords - Channel Assignment, Cellular Network, GA, SIR, Distributed Processing

\section{INTRODUÇÃO}

$\mathrm{O}$ avanço da telefonia celular resultou em um aumento na demanda desse tipo de serviço. Um dos tópicos que têm merecido atenção dos pesquisadores é a alocação de canais entre os terminais móveis, de modo que atenda a demanda de tráfego e a mínima SIR dos canais.

O processo de alocação de canais entre os usuários do sistema de comunicação móvel pode ser fixo, dinâmico ou híbrido [1]. Na alocação fixa, os canais são designados permanentemente para cada célula. Na alocação dinâmica, todos os canais estão disponíveis para todas as células e são assinaladas de acordo com a demanda. Alocação híbrida é uma mistura das técnicas de alocação fixa com a dinâmica, os canais podem ser alocados de forma fixa ou dinâmica. Assim, a questão a ser resolvida é obter uma alocação sem conflito de canais entre as células, satisfazendo a mínima SIR e a demanda de tráfego, otimizando a alocação de canais.

Uma abordagem interessante desse problema é o uso do algoritmo genético [2], [3] e [4]. Tal enfoque constitui-se em um ramo da inteligência artificial e baseia-se nos processos naturais de seleção, cruzamento e mutação genética, conhecidos como operadores genéticos [5]. Em [6] propõe uma alocação de canal com algoritmo genético distribuído e SIR, entretanto o trabalho analisa somente um estado e sem preocupação com

Ailton Akira Shinoda e Leandro Calixto T. de Albuquerque, Departamento de Engenharia Elétrica, Faculdade de Engenharia de Ilha Solteira, Universidade Estadual Paulista, Ilha Solteira, SP, Brasil, E-mails: shinoda@dee.feis.unesp.br, leandroalbuquerque@hotmail.com. o tempo para determinar a função de custo ideal. O trabalho em questão procura sanar essas deficiências.

$\mathrm{O}$ artigo está organizado na seguinte ordem. A seção II descreve o modelamento da alocação de canais considerando a demanda de tráfego e a mínima SIR. A seção III discute o algoritmo genético com uma breve revisão dos operadores genéticos. A seção IV descreve o modelamento da alocação de canais para o AG. A seção V apresenta um breve conceito da interface de passagem de mensagem (MPI) [7] pois para operar com AG paralelo, uma alternativa é utilizar vários processos que são alocados em processadores distintos e que precisam se comunicar através da troca de mensagens. A seção VI mostra os resultados obtidos de uma rede celular na alocação de canais, em uma simulação dinâmica, satisfazendo a demanda de tráfego com distribuição de Poisson, a mínima SIR e limitação de tempo com a técnica proposta.

\section{Modelamento Do CAnAL}

Considere uma rede celular constituída de $n$ células. Sem perda de generalidade, assuma que os canais estejam igualmente espaçados no espectro de rádio freqüência. Usando um mapeamento apropriado, canais podem ser representados como números inteiros positivos consecutivos.

A interferência ocorre quando a mesma freqüência é empregada simultaneamente por dois ou mais usuários na rede. Nesse trabalho estamos considerando as seguintes condições:

- Todas as células transmitem com a mesma potência;

- A perda de propagação é a mesma para todas as células;

- Todas as células possuem o mesmo tamanho.

A potência média recebida à uma distância $r$ da ERB (estação rádio base) é $P_{T} r^{-p}$, onde $P_{T}$ é a potência de transmissão da célula e $p$ a perda de propagação. Assim a SIR do canal $k$ alocado ao usuário da célula $i$ é dada por [8],

$$
S I R_{i}(k)=\frac{P_{T} r^{-p}}{\sum P_{i n t}}
$$

onde

- $S I R_{i}(k)$ é a SIR do canal $k$ da célula $i$;

- $r$ é a distância entre o usuário, alocado no canal $k$, e a ERB da célula $i$;

- $\sum P_{i n t}$ é a potência média recebida pela célula $i$ de todos os usuários das outras células que estão usando o canal $k$.

Portanto os valores da SIR podem ser descritos por uma matriz $n \times m$, onde $n$ é o número de células na rede e $m$ 
o número de canais, denominada de matriz de relação sinal interferência $\boldsymbol{S}$, onde cada elemento $s_{i j}$ fornece a SIR do canal $j$ da célula $i$. A demanda de tráfego pode ser expresso por um vetor $\boldsymbol{d}$ de $n$-elementos. Nesse vetor, cada elemento $d_{i}$ representa o número de canais requisitados para a célula $i$. Em resumo, dada a matriz de relação sinal interferência de $\boldsymbol{S}$ e o vetor de demanda $\boldsymbol{d}$, o esquema de alocação ótima de canal envolve a determinação do espalhamento mínimo de canal requerido e o meio de distribuir esses canais entre as células satisfazendo a mínima SIR e a demanda de tráfego. Seja o espaço de solução $\boldsymbol{F}$ como uma matriz binária $n \times m$, onde $n$ é o número de células e $m$ o número total de canais. Cada elemento $f_{j k}$ na matriz é um ou zero tal que

$$
f_{j k}=\left\{\begin{array}{l}
1 \text { (canal } k \text { está alocado na célula } j) \\
0 \text { (canal } k \text { não está alocado na célula } j)
\end{array}\right.
$$

Os requisitos básicos de uma rede celular são a capacidade de prover o tráfego e operar com um valor mínimo de qualidade. A primeira exigência impõe uma restrição de demanda sob a matriz $\boldsymbol{F}$. Um total de $d_{i}$ canais é requerido para a célula $i$. Isto implica que o número total de 1s na linha $i$ de $\boldsymbol{F}$ deve ser $d_{i}$. Matematicamente, isto significa que se a alocação de canais da célula $i$ violar a restrição de demanda, tem-se

$$
\left(\sum_{q=1}^{m} f_{i q}-d_{i}\right) \neq 0
$$

A segunda restrição é modelada por uma matriz de relação sinal interferência $S$. Se o canal $k$ da célula $i$ não estiver satisfazendo a mínima $\operatorname{SIR}\left(S I R_{i}(k)<S I R_{\text {min }}\right)$ então o canal $k$ não deve ser alocado para a célula $i$. Matematicamente, isto significa que se a alocação do canal $k$ na célula $i$ viola a restrição de mínima SIR, teremos

$$
\sum_{q=1}^{m} \operatorname{sgn}\left[S I R_{\text {min }}-S I R_{i}\left(q f_{i q}\right)\right]>0
$$

onde

$$
\begin{aligned}
& \operatorname{sgn}(x)= \begin{cases}1, & x>0 \\
0, & x \leq 0\end{cases} \\
& \operatorname{SIR}_{i}(0)=S I R_{\text {min }}
\end{aligned}
$$

Portanto, a seleção genérica da função de custo pode ser expressa como

$$
\begin{aligned}
C(\boldsymbol{F})= & \sum_{i=1}^{n}\left(\sum_{q=1}^{m} \operatorname{sgn}\left[S I R_{\min }-S_{I} R_{i}\left(q f_{i q}\right)\right]\right) \\
& +\sum_{i=1}^{n}\left(\sum_{q=1}^{m} f_{i q}-d_{i}\right)
\end{aligned}
$$

Assim, o problema é determinar $\boldsymbol{F}$ tal que $C(\boldsymbol{F})$ seja mínimo.

\section{Conceito do Algoritmo Genético}

$\mathrm{Na}$ evolução genética, processo iterativo do AG, o indivíduo mais apto (com maior valor de aptidão) tem a tendência de gerar como frutos, descendentes de boa qualidade, melhorando a cada geração o material genético da população (conjunto de indivíduos).

Numa aplicação prática de AG, precisa-se estabelecer uma população de indivíduos e estes podem ser iniciados com valores aleatórios. Em cada ciclo de operação, uma geração subseqüente é criada a partir dos cromossomos da população atual. Um grupo desses cromossomos, chamados de "pais", é selecionado por uma rotina específica (estratégia de seleção). Os genes dos pais são recombinados para a produção de descendentes na próxima geração. A partir disto, é esperado, deste processo evolutivo (manipulação de genes), que o "melhor"cromossomo criará o maior número de descendentes, e assim obtenha uma grande chance de sobrevivência nas gerações subseqüentes, simulando o mecanismo da seleção natural [5].

O ciclo de evolução é repetido até um determinado critério de término. Este critério pode ser o número de ciclos de evolução, ou a soma de variações de indivíduos entre gerações diferentes, ou um valor de custo pré-definido.

Duas operações fundamentais são utilizadas para facilitar o ciclo de evolução: cruzamento e mutação, embora a rotina de seleção ou reprodução possa ser considerada como outra operação.

$\mathrm{Na}$ operação de cruzamento, uma posição ao longo da sequiência é fixada aleatoriamente para cruzamento e as partes dos dois cromossomos além deste ponto serão trocadas para formar a descendência. Uma variável, chamada probabilidade de cruzamento $p_{c}$, é utilizada para controlar a frequiência de ocorrência do cruzamento, que na prática tem um valor típico entre $60 \%$ e $100 \%$.

No caso da mutação, a operação aplica-se individualmente a cada descendência após o cruzamento. Nesse mecanismo altera-se uma posição aleatoriamente com uma probabilidade de mutação $p_{m}$ que normalmente está abaixo de $1 \%$. Esta probabilidade $p_{m}$ é responsável pelo aumento da diversidade da população.

\section{Modelamento da Alocação de Canal em AG}

A Tabela I representa a configuração de uma rede celular, onde cada indivíduo é uma matriz $\boldsymbol{F}$ com células e canais alocados ( 0 indica canal não alocado e 1 indica canal alocado). A soma de 1's na linha indica a demanda de tráfego da célula em questão.

TABELA I

CONFIGURAÇÃo (CROMOSSOMO) DA REDE CELULAR

\begin{tabular}{|c|c|c|c|c|c|c|}
\hline \multirow{2}{*}{ cel } & \multicolumn{7}{|c|}{ canal } & \multirow{2}{*}{ Tráfego } \\
\cline { 2 - 6 } & 1 & 2 & 3 & $\cdots$ & $m$ & \\
\hline 1 & 0 & 1 & 0 & $\cdots$ & 0 & $d_{1}$ \\
\hline 2 & 0 & 0 & 1 & $\cdots$ & 1 & $d_{2}$ \\
\hline 3 & 0 & 0 & 1 & $\cdots$ & 0 & $d_{3}$ \\
\hline$\vdots$ & $\vdots$ & $\vdots$ & $\vdots$ & $\cdots$ & $\vdots$ & $\vdots$ \\
\hline$n$ & 1 & 0 & 0 & $\cdots$ & 1 & $d_{n}$ \\
\hline
\end{tabular}

Essa propriedade deve ser mantida durante todo o processo interativo do AG. A função de custo ou aptidão de $\boldsymbol{F}$ é calculada pela Eq.(4).

Considere o seguinte sistema celular com $n=4$ células ilustrado na Figura 1.

Além disso, o vetor de tráfego é $\boldsymbol{d}=[1,3,2,1]$ e $m=7$ canais disponíveis. Uma possível configuração ou indivíduo está ilustrado na Tabela II. 


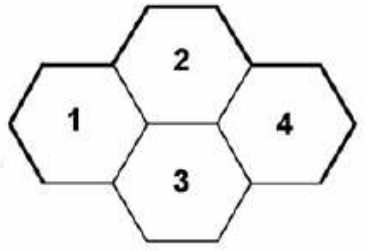

Fig. 1. Rede celular.

TABELA II

INDIVÍDUO OU CONFIGURAÇÃO DA REDE CELULAR DA FIGURA 1.

\begin{tabular}{|c|c|c|c|c|c|c|c|c|}
\hline \multirow{2}{*}{ cel } & \multicolumn{7}{|c|}{ Canal } & \multirow{2}{*}{ Tráfego } \\
\cline { 2 - 8 } & 1 & 2 & 3 & 4 & 5 & 6 & 7 & \\
\hline 1 & 0 & 0 & 1 & 0 & 0 & 0 & 0 & 1 \\
\hline 2 & 1 & 0 & 0 & 0 & 1 & 0 & 1 & 3 \\
\hline 3 & 0 & 1 & 0 & 1 & 0 & 0 & 0 & 2 \\
\hline 4 & 0 & 0 & 1 & 0 & 0 & 0 & 0 & 1 \\
\hline
\end{tabular}

\section{MPI}

Um sistema paralelo consiste em fazer com que o sistema operacional (no caso desse trabalho, GNU linux, distribuição Linux Fedora) possa compreender que um determinado software deva rodar paralelamente, e como ele deve ser distribuído. Para tanto, deve-se implementar as instruções que dizem como, quando e onde deve ser feita a divisão do software em processos menores que irão ser executados independentemente em cada um dos nós. Essa implementação é realizada dentro do próprio código fonte do software ( no caso dessa máquina, em MPI). No cluster empregam-se dois tipos de paralelização, a de grão grosso, que consiste na distribuição entre os diversos nós da máquina diferentes processos menores. E a de grão fino, que tenta paralelizar os laços e sub-rotinas dos softwares. O sistema foi projetado visando o funcionamento em um conjunto de PCs conectados numa rede local Ethernet 100 Mbps. A implementação foi realizada utilizando-se a linguagem $\mathrm{C}$, o padrão de troca de mensagens MPI para computação distribuída e o sistema operacional GNU Linux.

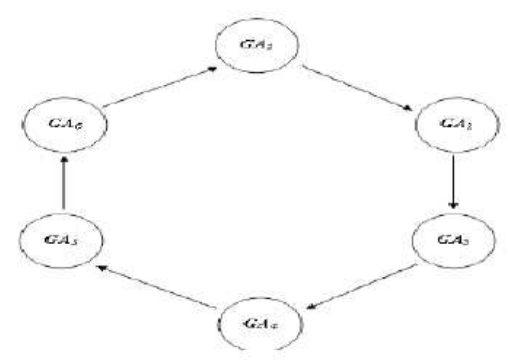

Fig. 2. Topologia em migração de anel.

Como o AG possui arquitetura intríseca paralela, não há um esforço extra para construir um modelo computacional paralelo. Dependendo da maneira em que o paralelismo é explorado na estrutura populacional e mecanismo de recombinação do AG, podemos classificar os métodos como: global, migração e difusão. Nesse trabalho optou-se pelo método de migração em anel, onde metade dos indivíduos de cada população é transferida entre sub-populações adjacentes unidirecionalmente, ilustrado na Figura 2.

\section{RESUltados}

A Figura 3 descreve a rede celular empregada nesse trabalho.

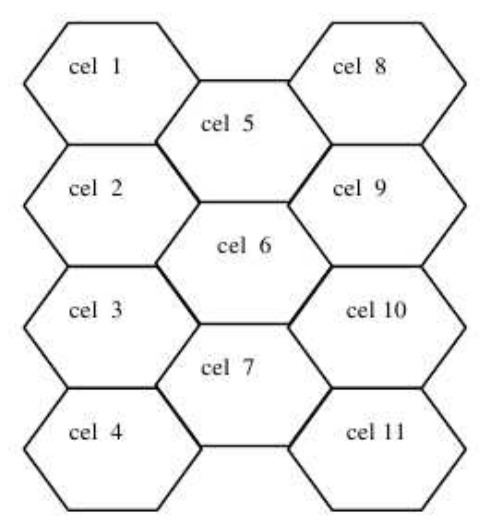

Fig. 3. Rede celular simulado.

A Tabela III informa a localização de cada ERB na rede para um raio de $1 \mathrm{~km}$ e o vetor da média da distribuição de Poisson de cada célula.

TABELA III

LocalizaÇÃo DA ERB E MÉdia DE Chamadas DA DistribuiçÃo DE POISSON.

\begin{tabular}{|c|c|c|c|}
\hline$n$ & $X(\mathrm{~km})$ & $Y(\mathrm{~km})$ & Média \\
\hline 1 & 1,00 & 6,06 & 6 \\
\hline 2 & 1,00 & 4,33 & 9 \\
\hline 3 & 1,00 & 2,60 & 9 \\
\hline 4 & 1,00 & 0,87 & 6 \\
\hline 5 & 2,50 & 5,20 & 9 \\
\hline 6 & 2,50 & 3,46 & 12 \\
\hline 7 & 2,50 & 1,73 & 9 \\
\hline 8 & 4,00 & 6,06 & 6 \\
\hline 9 & 4,00 & 4,33 & 9 \\
\hline 10 & 4,00 & 2,60 & 6 \\
\hline 11 & 4,00 & 0,87 & 9 \\
\hline
\end{tabular}

No caso dos usuários, assumiu-se uma distribuição espacial uniforme em cada célula. O número de usuários ou chamadas em cada célula é gerada pela distribuição de Poisson com as médias da Tabela III.

O algoritmo de alocação de canais AG distribuído está ilustrado na Figura 4. Cada processador do cluster (P0, P1, ..., Pn) executa o AG seqüencial. Após uma quantidade prédefinida de gerações internas, cada processador informa o valor da sua função de custo $(\mathrm{CF})$ ao processador mestre (P0). $\mathrm{O}$ processador mestre verifica se há algum CF nulo. Quando isso ocorre, o AG distribuído é encerrado. Se o $\mathrm{CF}$ não é nulo e a temporização não tenha encerrada é feita uma troca 
parcial de indivíduos entre os processadores (Figura 2), denominada de migração, iniciando uma nova interação. Quando há o término da temporização com CF diferente de zero, significa que o sistema está com bloqueio, ou seja, uma parte das chamadas não está sendo atendida. Diversos parâmetros

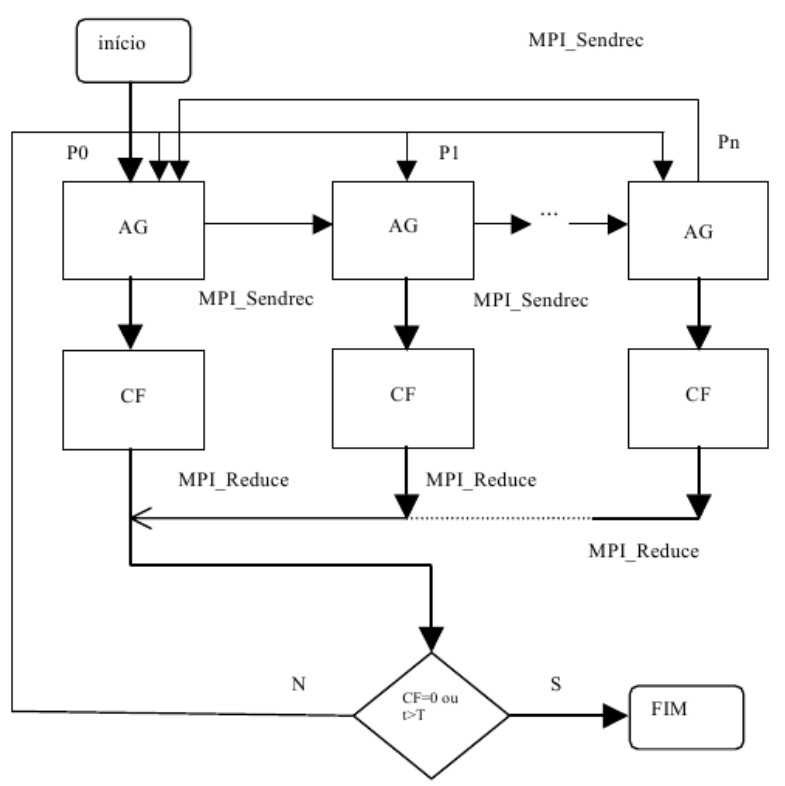

Fig. 4. Alocação de canais AG distribuído com temporização.

devem ser configurados, inclusive o número da população por processo $\left(N_{p}\right)$, a quantidade de canais disponíveis $(m)$, a taxa de cruzamento $\left(p_{c}\right)$, a taxa de mutação $\left(p_{m}\right)$, a taxa dos indivíduos menos aptos da população original $\left(p_{r}\right)$ serem incluídos na nova população, a taxa de migração $\left(p_{m g}\right)$, o número de gerações $\left(N_{G}\right)$ antes de uma nova migração, o raio da célula em $\mathrm{km}(R)$, a perda de propagação $(p)$, a mínima SIR aceitável em dB $(S I R)$ e o tempo máximo da busca ou temporização $(T)$ em segundos. Os valores utilizados nesse trabalho estão resumidos na Tabela IV.

TABELA IV

PARÂMETROS DA SIMULAÇÃO.

\begin{tabular}{|c|c|c|c|c|c|c|c|c|c|c|}
\hline$N_{p}$ & $m$ & $p_{c}$ & $p_{m}$ & $p_{r}$ & $p_{m g}$ & $N_{G}$ & $R$ & $p$ & sir & $T$ \\
\hline 16 & 48 & 0,5 & 0,01 & 0,2 & 0,5 & 50 & 1 & 3 & 10 & 1 \\
\hline
\end{tabular}

As simulações foram realizadas em um cluster de quatro computadores AMD 2,6 GHz, $512 \mathrm{Mb}$ de RAM interligados com comutador de 100 Mbps.

A Figura 5 ilustra as SIR's dos canais utilizados em cada uma das células para atender a demanda de tráfego, gerado através de uma distribuição de Poisson. A Figura 5 mostra somente as SIR' s de uma determinada condição ou estado do processo de Poisson.

A Tabela V mostra com maiores detalhes a Figura 5, onde a última linha é a demanda de tráfego ou chamada gerada pela distribuição de Poisson.

A Figura 6 mostra o desempenho do sistema, em termos da probabilidade de bloqueio, à medida que o valor médio da

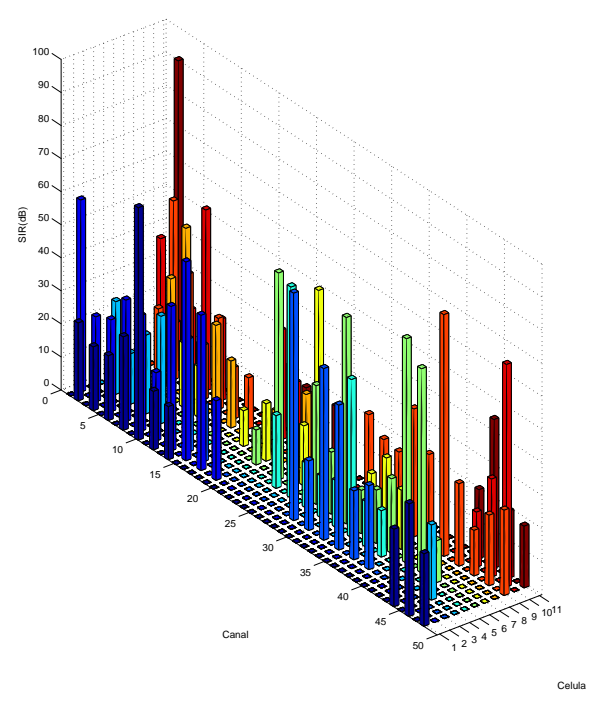

Fig. 5. Valor da SIR final (sistema sem bloqueio) para um determinado estado do sistema gerado pela distribuição de Poisson.

distribuição de Poisson (Tabela III) aumenta para o caso da alocação de canal AG distribuído com SIR limitado no tempo e também para o caso da alocação fixa de canais com re-uso de 4.

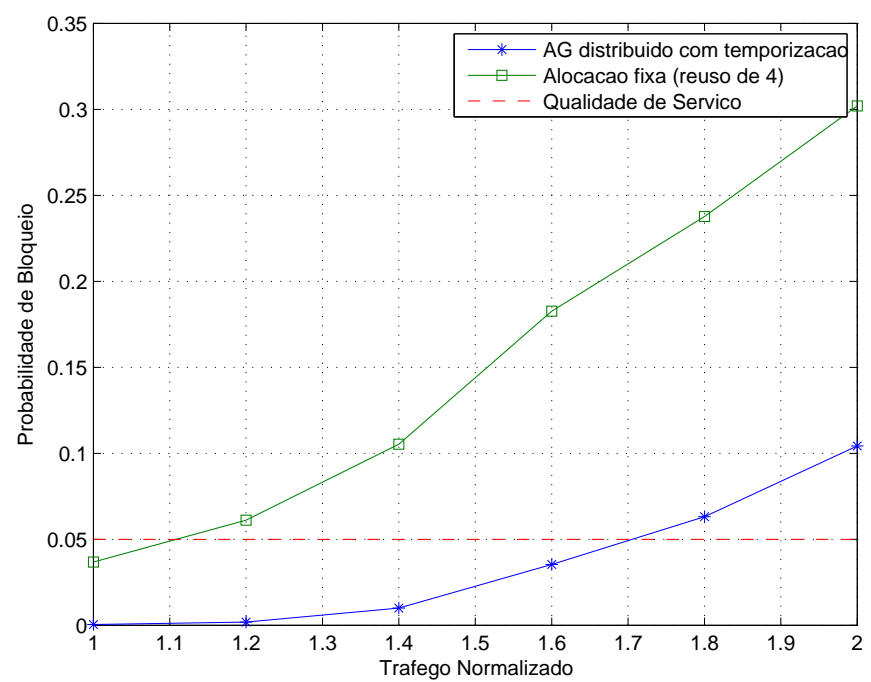

Fig. 6. Probabilidade de bloqueio do sistema em função do tráfego normalizado.

Cada ponto da curva foi calculado através de 100 estados ou condições geradas pela distribuição de Poisson com as médias da útima coluna da Tabela III multiplicadas pelo fator de normalização.

\section{CONCLUSÃO}

Foi apresentado um método alternativo de alocação de canal empregando AG distribuído e SIR com temporização. A 
técnica foi testada em mais de um estado (100 estados), com as chamadas em cada estado fornecidas por uma distribuição de Poisson. Nos resultados obtidos, o desempenho da técnica com processamento distribuído sempre foi superior em relação à alocação fixa. No caso específico do bloqueio em função do tráfego normalizado (Figura 6), enquanto a alocação fixa consegue atender um aumento de tráfego de apenas $10 \%$ o AG distribuído consegue atender um aumento de $70 \%$ para um grau de serviço de bloqueio de $5 \%$.

Diferentemente do que ocorre comumente com trabalhos de alocação de canais empregando técnicas evolutivas, onde não há limitação de tempo na busca do melhor conjunto de canais para atender as chamadas da rede celular, o trabalho apresenta essa limitação de tempo ou temporização.

\section{REFERÊNCIAS}

[1] R. Mathar and J. Mattfeldt, "Channel Assignment in Cellular Radio Networks", IEEE Transactions on Vehicular Technology, v. 42, pp.647656, 1993.

[2] S. S. M. Patra, K. Roy, S. Banerjee and D. P. Vidyarthi, "Improved Genetic Algorithm for Channel Allocation with Channel Borrowing in Mobile Computing”, IEEE Transactions on Mobile Computing, v.5, no.7, 2006.

[3] L. Wang, S. Arunkumar and W. Gu, "Genetic Algorithms for Optimal Channel Assignment in Mobile Communications", Proceedings of the 9th International Conference on Neural Information Processing, v.3, pp. 1221-1225, 2002.

[4] A. Y. Zomaya and M. Wright, "Observations on Using Genetic Algorithms for Channel Allocation in Mobile Computing", IEEE Transactions on Parallel and Distributed Systems, v.13, no.9, September 2002.

[5] D. Goldberg, Genetic Algorithms in Search, Optimization and Machine Learning. Addison-Wesley, 2nd Edition, New York, 1989.

[6] A. A. Shinoda e L. C. T. Alburquerque, "Alocação de Canais em Sistemas de Comunicação Celular Empregando Algoritmo Genético Distribuído e SIR", Anais do X Encontro de Modelagem Computacional, Nova Friburgo, RJ, 2007.

[7] G. E. Karniadakis, Parallel Scientific Computing in $\mathrm{C}++$ and MPI. Cambridge University Press, USA, 2003.

[8] M. Schwartz, Mobile Wireless Communications. Cambridge University Press, USA, 2006.
TABELA V

VALOR DA SIR FINAL.

\begin{tabular}{|c|c|c|c|c|c|c|c|c|c|c|c|}
\hline \multirow[t]{2}{*}{$m$} & \multicolumn{11}{|c|}{ célula } \\
\hline & 1 & 2 & 3 & 4 & 5 & 6 & 7 & 8 & 9 & 10 & 11 \\
\hline 1 & - & 58 & - & - & - & - & - & - & - & 37 & - \\
\hline 2 & 24 & - & - & - & - & - & - & - & 18 & - & 91 \\
\hline 3 & - & 26 & - & 28 & - & - & - & - & - & 15 & - \\
\hline 4 & 19 & - & - & - & - & - & - & - & 54 & - & - \\
\hline 5 & - & 28 & - & 15 & - & - & - & 33 & - & 22 & - \\
\hline 6 & 20 & - & - & - & - & - & - & - & 35 & - & - \\
\hline 7 & - & 37 & - & 24 & - & - & - & 52 & - & 55 & - \\
\hline 8 & 28 & - & - & - & - & - & 19 & - & 16 & - & - \\
\hline 9 & - & 35 & - & 32 & - & - & - & 19 & - & 25 & - \\
\hline 10 & 70 & - & - & - & - & - & 15 & - & 27 & - & - \\
\hline 11 & - & 21 & - & - & - & - & - & 28 & - & - & - \\
\hline 12 & 18 & - & - & - & - & - & - & - & 16 & - & - \\
\hline 13 & - & 44 & - & - & - & - & - & 20 & - & - & - \\
\hline 14 & 16 & - & 34 & - & - & - & - & - & 15 & - & - \\
\hline 15 & - & 60 & - & - & - & - & - & - & - & - & - \\
\hline 16 & - & - & 17 & - & - & - & 11 & - & - & - & \\
\hline 17 & - & 47 & - & - & - & - & - & - & - & 33 & - \\
\hline 18 & - & - & - & - & - & - & - & - & - & - & - \\
\hline 19 & - & 24 & - & - & - & 11 & 17 & 14 & - & 20 & 18 \\
\hline 20 & - & - & - & - & - & - & - & - & - & - & - \\
\hline 21 & - & - & - & - & - & - & - & 31 & - & 19 & 26 \\
\hline 22 & - & - & - & - & - & 63 & - & - & - & - & - \\
\hline 23 & - & - & - & - & 22 & - & - & 25 & - & - & 18 \\
\hline 24 & - & - & - & - & - & 13 & 18 & - & - & - & - \\
\hline 25 & - & - & - & - & 64 & - & - & - & - & - & - \\
\hline 26 & - & - & - & - & - & - & 62 & - & - & - & - \\
\hline 27 & - & - & - & - & 14 & 36 & - & - & - & - & - \\
\hline 28 & - & - & 69 & - & - & - & - & - & - & - & - \\
\hline 29 & - & - & - & - & 13 & 19 & - & - & - & - & - \\
\hline 30 & - & - & 21 & - & - & - & - & - & 28 & - & - \\
\hline 31 & - & - & - & - & 14 & 62 & - & - & - & - & - \\
\hline 32 & - & - & 52 & - & - & - & - & - & 24 & - & - \\
\hline 33 & - & - & - & - & 48 & 13 & 17 & - & - & - & - \\
\hline 34 & - & - & 44 & - & - & - & - & - & 23 & - & - \\
\hline 35 & - & - & - & - & 14 & 16 & 25 & - & - & - & - \\
\hline 36 & - & - & 21 & - & - & - & - & - & 39 & - & - \\
\hline 37 & - & - & - & - & 14 & 23 & 18 & - & - & - & - \\
\hline 38 & - & - & 25 & - & - & - & - & - & 28 & - & - \\
\hline 39 & - & - & - & - & - & 68 & - & - & - & - & - \\
\hline 40 & - & - & - & - & - & - & - & - & 73 & - & - \\
\hline 41 & - & - & - & - & - & 62 & - & - & - & - & - \\
\hline 42 & - & - & - & - & - & - & - & - & 25 & - & 21 \\
\hline 43 & - & - & - & - & - & 13 & - & - & - & 17 & - \\
\hline 44 & 23 & - & - & - & - & - & - & - & 14 & - & 45 \\
\hline 45 & - & - & - & 23 & - & - & - & - & - & 30 & - \\
\hline 46 & 34 & - & - & - & - & - & - & - & 21 & - & 20 \\
\hline 47 & - & - & - & - & - & - & - & - & - & 67 & - \\
\hline 48 & 22 & - & - & - & - & - & - & - & 26 & - & 19 \\
\hline$D$ & 10 & 10 & 8 & 5 & 8 & 12 & 9 & 8 & 17 & 11 & 8 \\
\hline
\end{tabular}

